\title{
Intricate partial waves in nuclear scattering
}

\author{
Heinrich Viktor von Geramb ${ }^{\mathrm{a}}$ \\ FB Physik, Universität Hamburg, Luruper Chaussee 149, 22761 Hamburg, Germany
}

Received: 30 September 2020 / Accepted: 13 October 2020 / Published online: 5 November 2020

(C) The Author(s) 2020

Communicated by Nicolas Alamanos

\begin{abstract}
This article is meant as an encomium of the lifespan endeavor of Jacques Raynal to relate nuclear scattering density matrices with numerical methods. The mathematical investigations he made involved intricate hypergeometric coupling algebras and transformations. He was among the first to appreciate the general importance of these studies. His efforts over 60 years are most praiseworthy and, for them, Jacques has gained much respect.
\end{abstract}

\section{Introduction and survey}

There are many branches in studies of nuclear reactions. Within those, a continuous effort has been to invent new tools with which to better understand regularities in the nuclear realm. Therein, however, pure quantum mechanical states are rather the exception. From the outset, scattering situations require measurement and interpretation of density matrices of the initial and final states involved. The usual approach, the $S$-matrix formalism, encompassed from the very beginning scattering models and analyses. In that formalism, initial and final states are connected by $S$-matrices which are deduced from nuclear transition $(T)$ matrices. A common approach to ascertain $S$-matrices is to solve Schrödinger's or even more advanced wave equations. To do so require sophisticated numerical methods to yield high speed and accurate results. In such developments, Jacques was a pioneer. As a result, today, we are well equipped with his programs to analyze data from very advanced, and often exotic, experiments. So common has this process become, that often its historical development seems to be forgotten. Current generations know much about the benefits of artificial intelligence (AI) and machine learning (ML) [1-3]. Being timely nowadays is often interpreted to mean that sciences, Physics in general,

The article carries the intention: Tribute to Jacques Raynal.

a e-mail: geramb@uni-hamburg.de (corresponding author)
Nuclear Physics and Nuclear Engineering in particular, are completely the province of AI/ML.

After 1950, Nuclear Physics was identified and lauded as an ultimate study for human benefit. Nuclear physics for peace was a paradigm everyone accepted. So universal was that belief that the IAEA, International Atomic Energy Agency, was founded on July 29 1957, as an autonomous specialized agency of the United Nations based in Vienna. The organization's task was to promote the peaceful use of nuclear energy, of cooperation and technology transfer. I remember participating as a scholar at the opening ceremony in the Konzerthaus in Vienna. The international importance of the subject lead, among many other initiatives, to foundation of the Abdus Salam International Center for Theoretical Physics (ICTP); an international research institute for physical and mathematical sciences that operates under a tripartite agreement between the Italian Government, United Nations Educational, Scientific and Cultural Organization, and the IAEA. An important initiative of the ICTP was to foster conferences and courses. The first in which I participated was the ICTP course Fundamentals in Nuclear Theory, Trieste 10/3-12/16 1966, IAEA Vienna (1967). On various occasions, Jacques Raynal and other members from CEA-Saclay were listed as speakers at ICTP seminars in Trieste.

This article starts when Jacques Raynal ${ }^{1}$ began his professional career at CEA-Saclay S.Ph.Math. in 1959/60, after 1963 S.Ph.Th. During the time when Jacques was a student at the elite school l'Ecole Polytechnique, he developed a high degree of proficiency in many topics, which in later years became manifest in his friendly 'Oh' style. My personal contacts with him span the years 1970-2006.

In his (and my) early years, given the importance attached to scientific effort world wide, many students including Jacques chose to make their professional life in studies of natural sciences and engineering. Many selected to do so

\footnotetext{
${ }^{1}$ Hereafter I will abbreviate his name with Jacques .
} 
in nuclear physics by working with computers in one way or another. At this time and later, CEA-Saclay acquired the most powerful main frame computing equipment from IBM, CDC and others; the intent to continue to do so persists to this day.

I'm not sure who coined the phrase computing as a language of physics. However, it may have been Lew Kowarski, a very remarkable personality associated with CEA-Saclay and CERN, who delivered a grand view lecture at the Seminar Course, ICTP-IAEA Trieste, August 1971, entitled: The impact of computers on nuclear science [4,5]. Also, Gordon Moore anticipated an increase of computing power by $2^{30}$ in 60 years; a change of 10 orders of magnitude. In comparison, the world population grew by a factor 2.5 in that time span. The surge in computing power helped drive productivity in all fields of Physics, nuclear physics especially. At the heart of the nuclear physics 'explosion' were use of the optical models. As a postdoc I counted 150 publications in Phys. Rev. having optical model or optical potential in their titles. Recently, I repeated the search with a wider journal range but the same phrases in the title, it gave significantly more than 15,000 papers.

As an aside, Jacques was important to me personally by his enabling my contact with many experts. For example, once when in Saclay, Jacques introduced me to the eminent scientist, Mme. Henriette Farragi, the random matrix expert, Madan Lal Mehta, the nucleon-nucleon $(N N)$ interaction experts at SATURN, and others.

As part of the survey, I briefly dwell upon inversion topics about which Jacques had a most supportive attitude. My interest in inversion theories began when I met Marcel Coz. He was once at the Institute de Physique Nucléaire, D.Ph.Th. in Orsay and later (1967) became a full Professor in theoretical physics at University of Kentucky. He collaborated, and often published, with Christine Coudray from the Institute de Physique Nucléaire in Orsay. They made use of Riemann functions, Marchenko inversion and closely related mathematics, which linked nuclear scattering with inverse problems at fixed angular momentum or fixed energy. Most skillfully, they were able to create generalized translation operators (GTO) and from a key paper entitled Existence of generalized translation operators from the Agranovitch-Marchenko transformation. Along with Marcel $\mathrm{Coz}$ and Christine Coudray the names, Levitan, Agranovich, Marchenko, Faddeev, Newton, and Sabatier [42-48] are often cited in reports on inversion mathematics and physics. Jacques, Marcel Coz, and Christine Coudray, along with many others, are testament to the excellent mathematical education and training in France. After 1976, we in Hamburg widened our view, went to topical conferences and workshops, and made effort in studies of inversion theories and applications.
The results are documented in dissertations and associated publications. We tackled quantum inversion theories by Gelfand-Levitan, Marchenko, and Darboux transformations, and numerically solved integral and algebraic equations for inversion of $N N$ data generating $N N$ inversion potentials. For energies, where the $S$-matrices are unitary, those inversion potentials are real. For non-unitary $S$-matrices complex two particle optical model inversion potentials were defined. In those studies, we used $N N$ phase-shift data from INS-DAC-Services SAID, George Washington University or from nn-online.org Radbound University Nijmegen $[49,50]$.

Using those inversion potentials, we solved LippmannSchwinger or better to say Bethe-Goldstone equations to generate reaction $g$-matrices which, notably, are energy and medium dependent. They belong to the class of effective complex energy and medium dependent interactions, and which are essential for many fields in nuclear theory [612]. Many ancillary studies supported our conjecture that hadron-hadron inversion potentials and/or general complex inversion optical potentials yield appropriate $g$-matrices as effective interactions that are explicitly energy and target medium dependent. An available optical model parameterization that yields agreement with phase shift data, is readily input into $g$-matrix calculations. I define them as two hadron inversion optical potentials. Andreas Funk [63], and colleagues before him, used primarily data from SAID or from nn-online.org. More on this subject is found in a later section. I have pursued this line of research until my retirement in 2003, after which, unfortunately, the evaluation and updating of inversion optical model potentials has been suspended.

Theoretical and technical aspects of $g$-matrix calculations are intricate. What is sought are medium and energy dependent effective interactions. The propagator in the defining equations should depend on all particle masses, charges, energies, momenta, angular momenta and spins. In solving such a complicated problem, use of hypergeometric functions could be practical and appropriate, as they have been in investigating three or four quasi-particle systems. Jacques was a leading expert in studies using hypergeometric functions.

In the next sections, Sects. 2 and 3, I emphasize aspects of Jacques 's work. Directly related with Jacques are: Fortran programs widely used for many analyses and in vogue to this day, generalized coupling schemes and relations with hypergeometric functions, generalized 3-j and 6-j symbols, and Moshinsky transformations [6-41]. In Sect. 3, the importance of the exceptional Raynal-Revei coefficients is discussed. Finally, in Sect. 4, more details on inversion and optical model hadron-hadron potentials are given. 


\section{Nucleon-nucleus scattering}

Jacques had an important role in this topic. In it he was influenced by the development of the helicity formalism of Jacob and Wick. Maurice René Michel Jacob (28 Mar 1933-May 2007) was a French theoretical particle physicist, he studied physics at l'Ecole Normale Supérieure from 1953 to 1957. During a visit to Brookhaven National Laboratory in 1959, he developed with Gian-Carlo Wick the helicity formalism for relativistic description of scattering of particles with spin and the decay of particles and resonant states. In 1961, he obtained a doctorate on this subject at the University of Paris. His thesis advisers were Professors Francis Perrin and GianCarlo Wick. Jacob then moved, as a post-doctoral fellow, to Caltech. He worked in Saclay from 1961 to 1967. Since 1967, he worked at CERN until his retirement in 1998.

In his first years in Saclay, Jacques solved a Schrödinger equation with factorization of radial and angular parts, by making use of the helicity formalism. He defined an optical model potential with real Coulomb, complex central and spin-orbit terms components. That optical potential model has now been used in many applications [9-12]. It is central in the widely used DWBA programs of Jacques (I do not recall the exact time when and where in Europe, I met Jacques and whether he or Richard Schaefer first told me about the development of DWBA70).

There are two theoretical and numerical features of note in the diverse set of DWBA codes (1970-2005, NEA 1209), as well as in all versions of ECIS (1970-2012, NEA 0850). They are the helicity formalism and the Numerov three point integration algorithm in solving second-order differential equations. A comprehensive and value rating of the Numerov algorithm can be found in the article by Melkanoff et al. [15].

Numerov's method, developed by the astronomer Boris Vasil'evich Numerov, is a numerical method to solve ordinary differential equations of second order in which a first derivative term does not appear. It is also known as Cowell's method. It is a fourth-order linear multi-step method that usually is implicit. It can be made explicit if the differential equation is linear. The helicity formalism, developed by Jacob and Wick, was for use with relativistic descriptions of scattering for particles with spin. For many reasons, this formalism is a must in high energy physics while a conventional formalism of nuclear physics uses the Wigner-Eckart theorem and elements of irreducible tensor algebra.

In Jacques 's codes, in which the radial step size is defined as $h$, the overall accuracy is set as $h^{4}$. This was deemed adequate for all practical situations in nuclear reaction calculations. Initially, computers with single precision 32 bits were sufficient to evaluate cross sections and other spin-dependent observables. Later 64 bits structures became standard and Jacques converted his programs from Fortran-77 to Fortran-
90/95, a useful tool for such conversion is from the pioneering work of M. Metcalf engendering the internet. Today, 128 bits and unlimited multi-precision Fortran is available. In 1990/95 Fortran became restructured and extended versions appeared. The latest version was formed in 2018.

\section{Generalized coupling and Raynal-Revai coefficients}

In $\mathcal{R}^{3}$ spherical coordinates, when taken as a polar coordinate system, involve a radius and two angles. In higher dimensions, $\mathcal{R}^{d}, d>3$, they are called hyperspherical coordinates, which comprise a hyperradius and $d-1$ hyperangles. In these coordinate systems, the angular momentum and irreducible tensor set re-coupling algebra are practitioners' tools, There are many studies of coupling and re-coupling coefficients [29,31-41] to show that the concepts of angular momentum in quantum physics, Racah-Wigner algebra, and $3-\mathrm{j}, 6-\mathrm{j}, 9-\mathrm{j}, 12-\mathrm{j}$ symbols, all fostered a new calculus that is far beyond that of classical angular momentum. The Clebsch-Gordan coefficients, (3-j symbols), belong to a set of six representations, six $3 F 2$, which suffices for 72 symmetries of the 3-j symbols, while Racah (6-j) coefficients belong to 4F3 and Saalschütz hypergeometric functions.

The news of the development of DWBA70 was a bit of a shock to me. I was totally unaware of it and had developed and written my own Fortran-IV code to analyze inelastic nucleon-nucleus scattering. That code called MEPHISTO was antisymmetrized and flexible with effective $N N$ interactions involving central, two-body spin orbit and tensor terms. This was a collaboration in the time span 1968 to 1970 with Ken Amos, who subsequently joined the University of Melbourne. At this time, Ken was an assistant Professor and I a three year visiting assistant Professor at the Physics department of the University of Georgia in Athens, Ga. In June 1970 I returned to Europe and was briefly with Amand Fässler in Münster, September 1970 to April 1971. Then, in May 1971 to March 1976, I had the chance to work in Jülich, in the newly established Amand Fässler Institut KFA-IKP-Theorie.

Another feature of the first meeting with Jacques was when he told me about his generalizations of 3-j and 6-j symbols in collaboration with János Révai, and more about use of hypergeometric coordinates. A key was Whipple's work on the symmetries of well-poised 7F6(1) and Saalschütz 4F3(1) series with unit argument applied to study the properties of 6-j symbols generalized to all arguments. For SU2, there are eleven different 4F3(1) series which can be used. Whipple's parameters provide a good description of symmetries and quite simple recurrence relations, valid for any arguments, can be obtained in terms of those parameters. Also, it was found that the transformation from one set of Jacobi coordinates to another for hyperspherical functions is closely related to the Talmi-Moshinsky transformations for two par- 
ticles in an oscillator well. The corresponding coefficients can be calculated analytically.

\section{Inversion and optical model hadron-hadron potentials}

This is a précis of our efforts and latest results which are contained in the thesis of Andreas Funk and in its publication in (2001).

Over many years GWU-SAID lists gave many nucleonnucleon phase shift solutions for low and medium energy regimes, and Arndt et al. have published many details of how those phase shifts are extracted from angular distributions. That SAID database has grown rapidly in the last three decades. While the proton-proton data now extends to $3 \mathrm{GeV}$, the neutron-proton data are limited to $1.3 \mathrm{GeV}$. Surprisingly, the solutions from SM97 to WI00 remain very closely the same and are very stable with regard to newly added data. We used the solutions SP00, FA00 and WI00 in calculations, finding results that differ only marginally. So, while most of our ensuing calculations are based upon SP00, the conclusions remain equally valid if other more recent data solutions would be used.

The GWU-SAID solutions [49] are parameterizations of the elastic channel, $N N, S$-matrices. For various reasons, we did not proceed directly with GWU-SAID rational interpolations. Instead we developed our own scheme for rational function representation of data and combined them with symmetric Padé approximants [8/8] or [10/10] of exp $(2 i x)$. This allowed contour integration of the Gel'fand-LevitanMarchenko integral equations, and thus assure precise radial inversion potentials pertinent for use in

$\left[-\frac{d^{2}}{d r^{2}}+\frac{\ell(\ell+1)}{r^{2}}+\frac{2 \mu}{\hbar^{2}} V_{\ell}(r)\right] \psi_{\ell}(r, k)=k^{2} \psi_{\ell}(r, k)$,

where $V_{\ell}(r)$ is local and energy independent. A simple substitution, and with $k^{2}=\lambda$, identifies this as a Sturm-Liouville equation,

$\left[-\frac{d^{2}}{d x^{2}}+q(x)\right] y(x)=\lambda y(x)$.

Solving Marchenko, Gel'fand-Levitan integral equations and Darboux transformations all yield principally the same solution and, numerically, they are complementary. In the Marchenko inversion, the input experimental information is the $S$-matrix, $S_{\ell}(k)=\exp \left(2 i \delta_{\ell}(k)\right)$, defining

$F_{\ell}(r, t)=-\frac{1}{2 \pi} \int_{-\infty}^{+\infty} h_{\ell}^{+}(r k)\left[S_{\ell}(k)-1\right] h_{\ell}^{+}(t k) d k$ which, when used in the Marchenko equation,

$A_{\ell}(r, t)+F_{\ell}(r, t)+\int_{r}^{\infty} A_{\ell}(r, s) F_{\ell}(s, t) d s=0$,

specifies the translation kernel $A_{\ell}(r, t)$. The inversion potential of Eq. (1) is a boundary condition for that translational kernel, namely

$V_{\ell}(r)=-2 \frac{d}{d r} A_{\ell}(r, r)$

Gel'fand-Levitan inversion has the same structure but uses Jost-functions which are related to the $S$-matrix. In the case of coupled channels, an integral equation suggested by Marcel Coz relates the $S$-matrix with the Jost-matrix.

In all practical applications rational functions are very appropriate to fit data within the unitary range, 0-300 MeV, with a simple extrapolation $1 / k$ towards infinity. For the range $0.3<T_{L a b}<3 \mathrm{GeV}$ we add an auxiliary complex energy dependent potential to explain that non-unitary regime. Initially, a local energy dependent auxiliary potential (Gaussian) served the purpose. In 2001, Andreas Funk replaced that local Gaussian by a set of symmetric separable Gaussian's. It is useful to use the Feshbach projection operator theory of optical potentials to discuss the process that was followed. In the Feshbach formulation, projection operators $P$ and $Q$ where $(P+Q)=1$, are conjectured. The operator $P$ is chosen to project into the unitary reference space, while inelastic and reaction channels are associated with $Q$ space. Using this, Andreas Funk distinguished three Hamiltonians, the reference Hamiltonian $H_{0}$, a projected Hamiltonian $H_{P P}$, and a full optical model Hamiltonian $\mathcal{H}$. With $H_{0}:=T+V_{0}$, the interaction $V_{0}$ is the unitary inversion potential. Using this he obtained solutions $\psi_{0}:=\psi_{0}^{+}(\mathbf{r}, \mathbf{k}, E)$ of $H_{0}$ from which a unitary reference $S$-matrix, $P H_{0} P=H_{P P}$, is defined. The $Q$ space is simplified by assuming it can be spanned by a minimum of states. Ultimately one or two harmonic oscillator functions with strengths, $\lambda_{i j}$, sufficed. Underlying resonances influence the values of those strengths. Thus, the full optical model Hamiltonian comprises the reference Hamiltonian $H_{0}$ and the optical model potential

$$
\begin{aligned}
\mathcal{V} & =\sum_{i j}\left|r, i>\lambda_{i j}<r^{\prime}, j\right|, \\
\mathcal{H} & :=T+V_{0}+\mathcal{V}\left(\lambda_{i j}, r, r^{\prime} ; l s j, E\right) .
\end{aligned}
$$

This Hamiltonian, $\mathcal{H}$, has regular solutions

$\Psi^{+}:=\Psi^{+}(r, k, l s j, E)$

whose asymptotic boundary conditions match the experimental elastic channel optical model potential $S$-matrix. The reference potential $V_{0}(r, l s j)$ and separable potential form factors were fixed, again by using $1 s$ and/or $2 s$ harmonic 
oscillator functions. The energy dependent strengths $\lambda_{i j}$ are uniquely determined with coordinate space reference potential Greens functions, the unitary $S_{0}(k)$ and full non-unitary $S(k)$.

Inote here a few applications using inversion and inversion optical model potentials. In 1996, Hugo Arellano, Francisco Brieva, Mathias Sander and I used $N N$ inversion potentials in calculations of $(\mathrm{p}, \mathrm{Ca})$ and $(\mathrm{p}, \mathrm{Pb})$ scattering cross sections taken at $500 \mathrm{MeV}$ incident energy. A microscopic optical model for nucleon-nucleus scattering was used. While the $N N$ inversion potentials implied alternative off-shell properties, when compared with those of meson theoretical models. The results of proton-nucleus elastic scattering in the 500 $\mathrm{MeV}$ region showed that otherwise often purported off-shell contributions are misleading. Better fits to data require, at the outset, accurate on-shell potentials, such as is assured with the generalized inversion potentials. A similar result was found by Martin Jetter from analyses of Bremsstrahlung data.

Hugo Arellano and I (2005) used $K N$ phase shifts to obtain Gel'fand-Levitan-Marchenko reference inversion potentials for those elementary systems. Those potentials were supplemented with a short range complex separable term in such a way that the corresponding unitary and nonunitary $K N S$-matrices were reproduced exactly. In the first lower momentum range the $K N$ real inversion potential (reference potential) enabled standard calculation of all on- and off-shell contributions for the $t$-matrices. In the second higher momentum range the $K N$ complex optical model potentials were used for the $t$-matrices and to determine the full-folding $K A$ optical model potentials. The beam momenta covered the range $400-1000 \mathrm{MeV} / \mathrm{c}$. The full folding optical model potentials gave agreement with elastic scattering data of $K A$ for ${ }^{6} \mathrm{Li},{ }^{12} \mathrm{C},{ }^{28} \mathrm{Si}$ and ${ }^{40} \mathrm{Ca}$. As this study included cases in which the elementary interaction implied a unitary $S$-matrix and the $K A$ optical potential many body inelasticities and reactions from the target alone. At higher momenta the $K N$ potential contains elementary complex reactions with a non-unitary $S$-matrix and $K A$ reactions are supplemented by many-body effects from the target nucleus. The study implied and confirmed nicely the transition from a unitary to a no-unitary $K N$ elementary interaction in $K A$ reaction cross sections. The elementary and many-body reaction routes don't interfere but simply add. The result was expected and the confirmation welcomed.

I am confident about the use of inversion mathematics and elementary phase shift data to investigate successfully certain topics in nuclear scattering. It is an effective data driven approach. As well as I wish Jacques Raynal to be remembered for his remarkable achievements, I hope our inversion approach, that he so kindly supported, will find others of like mind.

\section{Conclusion}

Jacques, merci.

Acknowledgements The author wishes to thank the referee for his constructive and stylistic suggestions to improve the manuscript.

Funding Open Access funding enabled and organized by Projekt DEAL.

Data Availability Statement This manuscript has no associated data or the data will not be deposited. [Authors' comment: I confirm, that this publication does not require associated data or a deposition. All information and results are either quoted in references, to my students akad. works, which is officially deposited in our Staats/Uni-Bibliothek in Hamburg and/or are published in quoted Journals/Books.]

Open Access This article is licensed under a Creative Commons Attribution 4.0 International License, which permits use, sharing, adaptation, distribution and reproduction in any medium or format, as long as you give appropriate credit to the original author(s) and the source, provide a link to the Creative Commons licence, and indicate if changes were made. The images or other third party material in this article are included in the article's Creative Commons licence, unless indicated otherwise in a credit line to the material. If material is not included in the article's Creative Commons licence and your intended use is not permitted by statutory regulation or exceeds the permitted use, you will need to obtain permission directly from the copyright holder. To view a copy of this licence, visit http://creativecomm ons.org/licenses/by/4.0/.

\section{References}

1. G.A. Negoita et al., Deep learning: a tool for computational nuclear physics, computational tools 2018: 9. Int. Conf. on Comp. Logic, Algebras, Programming, Tools and Benchmarks, 2018, Barcelona, IARIA (2019)

2. A.J.M. Plompen et al., The joint evaluated fission and fusion nuclear data library, FEFF-3.3. EPJ A56, 56-181 (2019)

3. S. Subair, C.H. Thorn, Implementations and Applications of Machine Learning, Studies in Computational Intelligence, vol. 782 (Springer, Berlin, 2020)

4. L. Kowarski, The impact of computers on nuclear sciences, in Computing as a language of physics, 27-37, ICTP Trieste 1971, IAEA Vienna SMR-9/8 (1972)

5. S. Fernbach, A. Taub (eds.), Computers and their Role in the Physical Sciences (Gordon and Breach, London, 1970)

6. H.A. Bethe, Selected works of Hans A (With commentary, World Scientific, Bethe, 1997)

7. G.E. Brown, C.-H. Lee, Hans Bethe and his Physics (World Scientific, Singapore, 2006)

8. S.S. Schweber, Nuclear Forces: The Making of the Physicist Hans Bethe (Cambridge University Press, Cambridge, 2012)

9. H. Feshbach, The optical model and its justification. Ann. Rev. Nucl. Sci. 8, 49 (1958)

10. H. Feshbach, Unified theory of nuclear reactions. Rev. Mod. Phys. 36, 1076 (1964)

11. H. Feshbach, The S-matrix for absorptive Hamiltonian. Ann. Phys. 165, 398 (1985)

12. H. Feshbach, Theoretical Nuclear Physics: Nuclear Reactions (Wiley, Hoboken, 1992) 
13. J. Raynal, Programme Fortran pour la diffusion élastique de deutons avec un modéle optique contenant des termes tensoriels, Report CEA-N-2287 (1963)

14. J. Raynal, Utilisation de faisceaux de deutons polarisés et détermination des paramétres du modéle optique, Thése, Faculté d'Orsay, June 24 1964, Report CEA-N-2511 (1964)

15. M.A. Melkanoff, T. Sawada, J. Raynal, Methods in computational physics. Nuclear Phys. 6, 1-80 (1966)

16. M. Jacob, G.C. Wick, On the general theory of collisions for particles with spin. Ann. Phys. 7, 404 (1959)

17. J. Raynal, Multipole expansion of a two-body interaction in helicity formalism and its applications to nuclear structure and nuclear reaction calculations. Nucl. Phys. A 97, 572-592 (1967)

18. J. Raynal, An iterative procedure for coupled channel calculations. Int. Symp. on Nuclear Structure, Dubna, Hongrie 4/7-11/7 1968, Report D-3893 (1968)

19. J. Raynal, MAGALI: a Fortran-IV program for automatic search in elastic scattering analysis with the nuclear optical model for spin 0,1/2 and 1 particles (Report S.Ph.Th, Saclay, 1969)

20. R. Schaefer, J. Raynal, DWBA 1970-2005, CEA-D.Ph.Th. Report R-4000, Saclay (1970)

21. J. Raynal, Calculations in nuclear physics, computing as a language of physics, ICTP Trieste 1971, IAEA Vienna SMR-9/8, 281-322 (1972)

22. J. Raynal, Spin-orbit interaction in the inelastic nucleon scattering, the structure of nuclei, ICTP Trieste 1971, IAEA Vienna SMR-8/8, 75-116 (1972)

23. J. Raynal, Aspects geometriques des reactions, contains Basel and Madison polarization conventions, Saclay S.Ph.Th. Note CEA-N1529, 1-102 (1972)

24. J. Raynal, Coupled channel calculations and computer code ECIS, Workshop on applied nuclear theory and nuclear model calculations for nuclear technology applications, ICTP Trieste 2/15-3/18 1988. World Scientific pp. 506-536, (1989)

25. J. Raynal, Notes on Ecis94-ec94.pdf, Report CEA-N-2772 (1994), and time dependent history of ECIS : Nishina Center for Accelerator-Based Science RIBF, ribf.riken.jp (2020)

26. D.G. Madland, A. J. Sierk, Development of global medium-energy nucleon-nucleus optical model potentials, Proc. Int. Conf. Nuclear Data for Science and Technology, ICTP Trieste , 5/19-24, 203 (1997)

27. A.J. Koning, J.-P. Delaroche, O. Bersillon, Nuclear data for accelerator driven systems: nuclear models, experiments and data libraries. Nucl. Inst. Meth. Phys. A414, 49 (1998)

28. M. Metcalf, J. Reid, M. Cohen, Fortan 95/2003 explained, Oxford (2004) [Modern Fortran Explained, Fortran 2018, Oxford (2018)]

29. T. Regge, Nuovo Cim. 10, 544 (1958)

30. T. Regge, Nuovo Cim. 11, 116 (1958)

31. A. De Shalit, I. Talmi, Nucl. Shell Theory Acad. 1963, 573 (1963)

32. J. Raynal, J. Revai, Transformation coefficients in the hyperspherical approach to the three body problem. Nuovo Cim. A $\mathbf{6 8}, 612-622$ (1970)

33. J. Raynal, Infinitesimal transformation for hyperspherical and Moshinsky functions. Nucl. Phys. A 202, 631 (1973)

34. J. Raynal, 3-j, 6-j and 9-j symbols, Report CEA-S.Ph.Th. (1973)

35. J. Revai, J. Raynal, Three body treatment of binding effects in multiple-scattering theory. Lett. Nuovo Cim. 9, 461-463 (1974)

36. J. Raynal, J. Revai, Recurrence relations between transformation coefficients of hyperspherical harmonics and their application to Moshinsky coefficients. Nucl. Phys. A 259, 272 (1976)

37. J. Raynal, On the various formulae for generalized 3-j symbols. (Int. Conf. on Nuclear Structure, Tokyo, 1977)

38. J. Raynal, On the definition and properties of generalized 3-j symbols. J. Math. Phys. 19, 467 (1978)

39. J. Raynal, On the definition and properties of generalized 6-j symbols. J. Math. Phys. 20, 2398-2415 (1978)
40. L.C. Biedenharn, J.D. Louck, Angular momentum in quantum physics, Vol. 8, and the Racah-Wigner algebra in quantum theory, Vol. 9, Encyclopedia of Mathematics and its Applications, Addison-Wesley (1981)

41. K. Srinivasa-Rao, Special topics in the quantum theory of angular momentum. Prama 24, 15-26 (1985)

42. V. Bargmann, On the connection between phase shifts and scattering Potential. Rev. Mod. Phys. 21, 488 (1949)

43. M.G. Krein, On integral equations generating differential equations of second order. Dokl. Akad. Nauk. SSSR 97, 21 (1954)

44. M. Coz, Ch. Coudray, Existence of generalized translation operators from the Agranovitch-Marchenko transformation. J. Math. Phys. 14, 1574 (1973)

45. M. Coz, The Riemann solution and the inverse quantum mechanical problem. J. Math. Phys. 17, 888 (1976)

46. I.V. Poplavskii, Generalized Darboux-Crum-Krein Transformations. Th. Math. Phys. 69, 1278 (1986)

47. K. Chadan, P.C. Sabatier, Inverse Problems in Quantum Scattering Theory (Springer, Berlin, 1989)

48. R. Pike, P. Sabatier, Scattering: Scattering and Inverse Scattering in Pure and Applied Science, I/II 958/1831 (Academic, Cambridge, 2002)

49. W. Briscoe, M. Doering, H. Haberzettl, I. Strakovsky, R. Workman (Staff), SAID Partial-Wave-Analysis facility, INS, George Washington University, Washington DC (2020)

50. Radbound University Nijmegen, nn-online.org (2020)

51. T. Kirst, NN Potentiale aus der Inversion, Diss. Hamburg (1989)

52. T. Kirst, NN Potentiale aus der Inversion. Phys. Rev. C 40, 912 (1989) (deceased (1992))

53. M. Jetter, NN potentials and there test with Bremsstrahlung, Diss. Hamburg (1994)

54. M. Jetter, NN potentials and there test with Bremsstrahlung. Phys. Rev. C 49, 1832 (1994)

55. H. Kohlhoff, Rationale Darstellung der NN S-Matrix, Dipl. Hamburg (1989)

56. H. Kohlhoff, NN Potentiale und Quanteninversion der Streumatrix, Diss. Hamburg (1993)

57. H. Kohlhoff, NN Potentiale und Quanteninversion der Streumatrix, Lect. Notes in Physics, pp. 427, Springer (1994)

58. L. Jäde, Consistent description of $\mathrm{NN}$ and (pi,N) interactions using the solitary boson exchange potential, Diss. Hamburg (1998)

59. L. Jäde, Consistent description of $\mathrm{NN}$ and (pi, $\mathrm{N}$ ) interactions using the solitary boson exchange potential. Phys. Rev. C 57, 496 (1998). (Phys. Rev. C 58 (1998))

60. L. Jäde, Consistent description of $\mathrm{NN}$ and (pi, N) interactions using the solitary boson exchange potential. Phys. Rev. C 1998, 58 (1998)

61. M. Sander, (pi,pi), (K,pi) and (pi,N) potential scattering and a prediction of a narrow sigma meson resonance, Diss. Hamburg (1997)

62. M. Sander, (pi, pi), (K, pi) and (pi, N) potential scattering and a prediction of a narrow sigma meson resonance. Phys. Rev. C 56, 1218 (1997)

63. A. Funk, NN optical model for energies up to $3 \mathrm{GeV}$, Diss. Hamburg (2001)

64. A. Funk et al., Phys. Rev. C 64, 054003 (2001)

65. H.F. Arellano et al., Phys. Rev. C 54, 2570 (1996)

66. H.F. Arellano, H.V. von Geramb, Extension of the full-folding optical model for nucleon-nucleus scattering with applications up to 1.5 GeV. Phys. Rev. C 66, 024602 (2002)

67. H.F. Arellano, H.V. von Geramb, Microscopic analysis of K+ nucleus elastic scattering based on $\mathrm{K}+$ nucleon phase shifts. Phys. Rev. C 72, 025203 (2005)

68. H.V. von Geramb (Ed.), Microscopic optical potentials, Lect. Notes in Physics 89, Springer (1979)

69. H.V. von Geramb (Ed.), Medium energy nucleon and antinucleon scattering, Lect. Notes in Physics, pp. 243, Springer (1985) 
70. H.V. von Geramb (Ed.), Quantum inversion theory and applications, Lect. Notes in Physics, pp. 427, Springer (1994)

71. B. Apagyi, G. Endrédi, P. Lévay (Eds.), Inverse and algebraic quantum scattering theory, Lect. Notes in Physics, pp. 488, Springer (1996)

72. H. Leeb, Exactly solvable models for the Schrödinger equation from generalized Darboux transformations. J. Phys. A 26, 5145 (1993)

73. J. Bystricky, F. Lehar, P. Winternitz, J. de Physique 39, 1 (1978) [NN Phase Shift Analysis, J. de Physique 48, 199 (1987)]
74. J. Arvieux, Phys. Saturn Z Phys. C Particl. Fields 46, 123-132 (1990)

75. R.A. Arndt, J.S. Hyslop III, L.D. Roper, Nucleon-Nucleon partial wave analysis to $1100 \mathrm{MeV}$. Phys. Rev. D 35, 128 (1986)

76. R.A. Arndt, J.S. Hyslop III, L.D. Roper, Nucleon-Nucleon partial wave analysis to $1.6 \mathrm{GeV}$. Phys. Rev. D 45, 3995 (1992)

77. R.A. Arndt, J.S. Hyslop III, L.D. Roper, Updated analysis of NN elastic scattering to 1.6 GeV. Phys. Rev. C 50, 2731 (1994) 